\section{Dementia in the old age: a gloomy wood later in life}

\author{
Virginia Boccardi, Lucia Paolacci, \\ Patrizia Mecocci
}

Institute of Gerontology and Geriatrics, Department of Medicine, University of Perugia, Italy

\begin{abstract}
Dementia incidence is growing at an impressive rate worldwide, mostly affecting old age subjects. Looking and considering the disease as the same in younger adult does not seem the successful way to find a proper solution regarding prevention and therapy this since there are too many differences between these two forms from biological to clinical aspects. Three question arises from a deep reflection on dementia in the oldest old: i) if it is a continuum with physiological brain aging; ii) what are the linking mechanisms underlining the disease and brain normal aging; iii) if or how it is possible to prevent or manage the disease differently in this population. We strongly believe that dementia is not an inevitable result of ageing, but when it appears in the oldest olds, it assumes distinctive characteristics of a geriatric syndrome where etiology, pathogenesis, clinical manifestations, course of the disease and management require a patient-tailored approach that can not be separated from a careful multidimensional evaluation.
\end{abstract}

\section{Introduction}

In the midway of this our mortal life, I found me in a gloomy wood, astray Gone from the path direct...

\section{Dante Alighieri, The Divine Comedy translated by Henry Francis Cary}

Age remains the most important risk factor for dementia and, along with the aging of the population worldwide, prevalence and incidence of the disease are growing at an impressive rate. Dementia is an umbrella term used to describe a group of symptoms characterized by a loss of cognitive and social functioning as well as behavioral changes. The prevalence of Alzheimer's disease (AD), the most common cause of dementia, is estimated of 48 million of persons in the world ${ }^{1}$ and the most of people affected are the so-called oldest olds, i.e. those aged 85 years or more. Approaching dementia later in life, clinicians can immediately appreciate the sense of impotence given by the complexity of such a disease, which assumes in this population the distinctive characteristics of a geriatric syndrome. Everything is complex in the oldest old: identification, diagnosis, management, treatment. Distinguishing brain aging from dementia is a hard job. Clinicians might experiment with the feeling of being lost, such as in a gloomy wood, considering how distinctive and different dementia is in the oldest as compared to the youngest subjects. The current research looks like a race against time to gain an early diagnosis with, unfortunately, no satisfying efficacious treatment at the moment. Furthermore, we are forgetting that, even if the oldest old represent the largest and fastest growing population with dementia, the majority of research efforts are still mainly focused on the younger population. Thus, an improved understanding of the physiopathological basis of dementia in this oldest population is of urgent and great importance.

\section{Brain aging and dementia: exploring the connections}

Aging is defined as the progressive decline in physiological functions which leads to increased vulnerability to diseases and ultimately to death. With advancing age, the majority of people experience declines in their ability to learn and recall new information. However, in healthy aging, these changes are very different from dementia, along with their neurobiological correlates. Understanding the nature of these cognitive changes as well as associated brain alterations represents a novel starting point for preventive and potential curative strategies. The nature of the relationship between brain aging and dementia must be identified in the biological peculiarity of the brain: an organ consisting of perennial cells (neurons), almost unable to regenerate and without the possibility of replacement in old age. Neurons are strongly connected to each other: it has been estimated that the human brain has about $10^{11}$ neurons connected by $10^{14}$ synapses. $^{2}$ Therefore, every single neuron can reach about one thousand connections with the others and even if there was a hypothetical neuron turnover, it would restore, for each neuron, all the pre-existent links to avoid the loss of neuron function. This seems quite complex and tricky to happen. Thus, the advantage of a post-mitotic system is
Correspondence: Virginia Boccardi, Institute of Gerontology and Geriatrics, Department of Medicine, University of Perugia, piazzale

Gambuli 1, 06132 Perugia, Italy.

Tel.: +39.0755783524.

E-mail: virginia.boccardi@unipg.it

Key words: Alzheimer's disease; brain aging; cognitive impairment; dementia; oldest old.

Conflicts of interest: the authors declare no potential conflict of interests.

Received for publication: 9 August 2018. Accepted for publication: 16 November 2018.

This work is licensed under a Creative Commons Attribution-NonCommercial 4.0 International License (CC BY-NC 4.0).

(C) Copyright V. Boccardi et al., 2018

Licensee PAGEPress, Italy

Geriatric Care 2018; 4:7756

doi:10.4081/gc.2018.7756

that the information acquired over time persists throughout life as stored as memory. The disadvantage consists in the fact that, as the system becomes old and damaged, its perfect and powerful functional capacity inevitably declines. Just like a last-generation phone, where the complexity and the high quality of its performance make it a delicate and sensitive tool over time.

As all our tissue, organs, systems and whole body, the brain during life undergoes cellular variations linked to aging, where cellular senescence represents the central element and the core feature of the so-called age-related changes (ARCs). ${ }^{3-6}$ Altogether, these changes can be considered as the result of an energy failure, leading neurons and other brain cells to a progressive decline, which ultimately limits the functional brain capacity. The decline of energy, mainly due to mitochondrial dysfunction, causes an increased entropy (defined as a measure of disorder according to the second law of thermodynamics). We can image that a cell is similar to a factory. The factory has a multitude of machines that metabolize food, repair DNA and dispose of garbage. The operators of these machines are the factory's workers-proteins. The industry is powered by chemical energy, which is provided by the mitochondria. However, like any chemical power source, it leaves behind unwanted waste, and when that is not disposed of properly, it will accumulate and damage the industry's functioning. Over time, cells lose their ability to function correctly, causing damaged or dead cells to accumulate in our body.

Thus with aging, the biological process- 
es that normally keep up brain structure and function decline inevitably, and altered misfolded proteins start to accumulate (amyloid, tau, $\alpha$-synuclein, TDP-43 and so on). ${ }^{7}$ ARCs determine a series of structural changes characterized, at the microscopic level, by the reduction of the number of neurons, dendritic arborizations and synaptic buttons as well as an increase of glial cells, especially in the cortical and extracellular space. All these changes lead to macroscopic changes such as reduction: i) of total brain weight and volume; ii) thickness of the prefrontal cortex; iii) hippocampal volume; and iv) an increase of the liquor cavities. Altogether, these changes affect the functional level. The processing speed (such as automatically performing easy tasks), the working memory (use the newly learned information such as a telephone number) as well as the episodic memory (keep and retrieve recent information, such as the shopping list) progressively reduce along with aging. On the contrary, the ability to use information related to one's autobiography and common knowledge, known as historical memory, strongly maintains or improves over time. Just what makes an old person wise. However, even if the brain aging represents an inevitable process in our life, something happens in some people who reach 90 years without dementia. If we think that Sophocles wrote Oedipus in Colonus when he was 89 years old, during a time in which the lifespan was surely shorter than now, two important issues come to light: the need to better understand the resistance mechanisms as well as preventive, strategies for dementia even, later in life.

\section{Resistance mechanisms: the reserve}

Dementia in the oldest olds is a still barely explored landscape made of different characteristic compared to young olds regarding pathogenesis and clinical presentation. While the majority of young patients with any kind of dementia show pathology commensurate with the severity of their cognitive impairment, in the oldest olds many individuals with abundant brain pathology are cognitively normal. For example, about $20 \%$ to $30 \%$ of cognitively normal elderly have a substantial amyloid deposition in the brain comparable to the levels observed in AD. ${ }^{8}$ To further complicate the matter, neuritic plaques also occur in not demented old age subjects. ${ }^{9}$ These observations suggest that the brain can tolerate a high damage accumulation without cognitive dysfunction. Interestingly, the brain, in the context of structural changes, puts into effect compensatory mechanisms characterized by: i) plasticity, or the ability to increase, after appropriate stimulation, the dendritic arborization, managing to keep active the different neuronal circuits; and ii) redundancy or the availability of circuits neuronal accessories that can substitute the main one when irreparably altered. These mechanisms express the ability of the senile brain to guarantee performances not substantially different from those of the young brain, as it can compensate for structural losses with the activation of a greater number of neuronal circuits. In fact, functional imaging studies have shown that older people show more elaborate brain activation than younger controls when performing the same motor tasks. ${ }^{10,11}$ These compensatory mechanisms are the basis for the brain reserve or resilience. However, this model presupposes the existence of a fixed limit, which, once reached, inevitably announces the appearance of clinical manifestations of cognitive decline. Interestingly, a study from an Israeli group showed a pattern of reduced functional connectivity (as evaluated by functional magnetic resonance imaging) in highly functioning nonagenarians during a recognition memory task, as compared to younger subjects. ${ }^{12}$ This finding suggests the existence also of the cognitive reserve, indicating the existence of neuropathological damage resilience, defined as the ability to optimize and maximize performance. The brain can be imagined as a large-scale network, not much different from many social or technological networks. Just like social networks, brain networks contain subnets or systems of highly correlated or interacting nodes (in the case of the brain, nodes can represent neurons or brain areas). Along with aging, there is a decrease in the segregation of brain systems, ${ }^{10}$ which reflects the degree to which they share inter-connections closely related to the ability of cognitive functions. This is a model of efficiency, and it implies that the task is processed with fewer resources until the connections are completely lost. The cognitive reserve represents what is acquired, learned throughout life by all past experiences. In a few words, it represents the amount of highly correlated or interacting nodes that we were able to build during life. It is possible to conjecture that highly performing oldest old could build more close connection and nodes. Several factors that predict a lower risk of dementia, including high-quality education, occupational complexity, and balanced diet, were also associated with the biological advantage of cognitive reserve. ${ }^{13}$ According to this hypothesis, elderly people with a high level of cognitive reserve may remain dementia-free in spite of cerebral neuropathological changes. Using an analogy, brain reserve can be seen as the hardware, while cognitive reserve like the software of our brain-mind system. Therefore, the interindividual differences observed in the course of aging would find their origin both in the brain at the structural level (individuals endowed with a more important number of neurons or synapses would benefit from a higher cerebral reserve); and at the functional level (the efficacy, the capacity and the flexibility of the available networks of functional connections between neurons, or of the cognitive processes assigned to the processing of information, would allow asking for networks of connections or alternative cognitive processes to compensate the damages due to pathology).

\section{Prevention strategies of dementia later in life}

While there is a broad consensus on the need to develop drugs specific for prototypical cognitive decline in young old, the mosaic nature of the molecular and cellular neuropathology in the oldest old makes harder the efficacy of a tailored drug. For its nature, dementia in the elderly may represent an advantage for the clinicians in taking advantages from peculiar prevention or cognitive stimulation strategies to boost neuronal plasticity and brain resilience. ${ }^{14}$ Acting on dementia prevention, intervention, and care will improve living for individuals affected and their families. Pathological cognitive decline is not an inevitable consequence of aging, and there are potentially modifiable health and lifestyle factors from different phases of life that, if corrected. Collectively it is never too late to reduce dementia risk. People born more recently have a lower risk to suffer from such a disease than who born earlier in the past century and the scale of the reduction identified is in line with major reductions in risk factors, such as higher levels of education and health satus. ${ }^{15}$ Modifiable risk factors changes during life. For example, a low educational level in early life results in lower cognitive reserve. Many factors increase dementia prevalence (hypertension, obesity, vascular incidents as well as survival after stroke and multimorbidity) mainly acting in the middle age. Even if, cardiovascular risk factors in adult life have a prevalent role in determining dementia in the oldest olds, but their relationship in the oldest old is not as linear as compared in the young old. Surprisingly, in the oldest-old late-life hypertension ${ }^{16,17}$ seems to be protective for dementia while other factors such as diabetes, smoking, depression, lack of physical exercise and social isolation maintain a decisive role. ${ }^{17}$ Another common risk factor for cognitive decline is the age-related neurosensory loss 
that affects more than half individuals aged over 75 years. ${ }^{18}$ Notably, several studies showed that association between presbycusis and cognitive impairment was not the consequence of the sole poor verbal communication, that can confound cognitive tests, but it was also maintained when using nonverbal cognitive tests ${ }^{19}$ and that interventions that restore hearing might be effective for alleviation of late-life cognitive disorders. ${ }^{20}$ Indeed, polypharmacy, strongly linked to multimorbidity, plays a silent and sneaky role with special regard to those medications with anticholinergic properties: antiemetics, antispasmodics, bronchodilators, antiarrhythmic drugs, antihistamines, analgesics, antihypertensive, antiparkinsonian agents, corticosteroids, skeletal muscle relaxants, and psychotropic drugs. A large metanalysis showed that exposure to anticholinergic drugs might increase not only the risks of cognitive impairment but also the risk of falls and all-cause mortality in the oldest olds. ${ }^{21}$

\section{Conclusions and final remarks}

The number of people with dementia is increasing globally and in particular in older populations. In the presence of an oldest old with cognitive impairment, it is essential to consider that multiple conditions may be able to take part in its expressiveness or in itself induce it. In this context, the patient approach cannot be separated from a careful multidimensional evaluation. In particular, the comorbidity can be used as a therapeutic opportunity, the one that represents the geriatric paradox. To improve cognitive functions, people with dementia should be offered cholinesterase inhibitors or memantine. Unfortunately, very old patients often do not yield to therapy. Younger age is associated with better anatomical and functional conditions of the brain that may positively act a better shortterm effect of treatment, ${ }^{22}$ but exclusion from drug prescription based on age is unappropriated. In fact, it has been demonstrated that the oldest age is associated with a slower progression of the disease independently of the initial response during the next years of treatment as compared with subjects with a first positive response. ${ }^{23}$ Another crucial issue is the social and family support to these patients. Family carers are at high risk of depression and most importantly, in the aged world of dementia, having an aged caregiver is very common. The family is still considered essential to guarantee the quality of life of people with dementia, for whom home is the best and preferred care setting. A failure in caregiver health may collapse this fragile support system. People with dementia and society need protection from possible risks of the condition, including self-neglect. A third of older people die with dementia, so it is essential that professionals working in end-of-life care consider whether a patient has dementia, because they might be unable to make decisions about their care and treatment or express their needs and wishes. Indeed, technological interventions have the potential to improve care delivery but should not replace social contact.

Altogether, the geriatric approach might be the way to exit from the gloomy wood and see even by little, the light beyond the trees.

\section{References}

1. Prince M, Comas-Herrera A, Knapp, M, et al. World Alzheimer report 2016: improving healthcare for people living with dementia: coverage, quality and costs now and in the future. London: Alzheimer's Disease International (ADI); 2016.

2. Williams RW, Herrup K. The control of neuron number. Annu Rev Neurosci 1988; 11:423-53.

3. Drachman DA. Aging of the brain, entropy, and Alzheimer disease. Neurology 2006;67:1340-52.

4. Xu J, Kobayashi S, Yamaguchi S, et al. Gender effects on age-related changes in brain structure. Am J Neuroradiol 2000;21:112-8

5. Park DC, Bischof GN. The aging mind: neuroplasticity in response to cognitive training. Dialog Clin Neurosci 2013;5: 109-19.

6. Chinta SJ, Woods G, Rane A, et al. Cellular senescence and the aging brain Exp Gerontol 2015;68:3-7.

7. Mecocci P, Baroni M, Senin U, Boccardi V. Brain aging and late-onset Alzheimer's disease: a matter of increased amyloid or reduced energy? JAD 2018;64:S397-404.

8. Aizenstein HJ, Nebes RD, Saxton JA, et al. Frequent amyloid deposition without significant cognitive impairment among the elderly. Arch Neurol 2008;65: 1509-17.

9. Monsell SE, Mock C, Roe CM, et al. Comparison of symptomatic and asymptomatic persons with Alzheimer disease neuropathology. Neurology 2013;80:2121-9.

10. Chan MY, Park DC, Savalia NK, et al. Decreased segregation of brain systems across the healthy adult lifespan. Proc Natl Acad Sci U S A 2014;111:E49975006.

11. Wang TH, Kruggel F, Rugg MD. Effects of advanced aging on the neural correlates of successful recognition memory. Neuropsychologia 2009;47:1352-61.

12. Beeri MS, Lee $H$, Cheng $H$, et al. Memory activation in healthy nonagenarians. Neurobiol Aging 2011;32:515-23.

13. Kravitz E, Schmeidler J, Beeri MS. Cognitive decline and dementia in the oldest-old. Rambam Maimonides Med J 2012;3:e0026.

14. Mattson MP. Late-onset dementia: a mosaic of prototypical pathologies modifiable by diet and lifestyle. Aging Mechan Disease 2015;1:15003.

15. Matthews FE, Arthur A, Barnes LE, et al. A two-decade comparison of prevalence of dementia in individuals aged 65 years and older from three geographical areas of England: results of the Cognitive Function and Ageing Study I and II. Lancet 2013;382:1405-12.

16. Assuncao N, Sudo FK, Drummond C, et al. Metabolic syndrome and cognitive decline in the elderly: a systematic review. PLoS One 2018;13:e0194990.

17. Livingston G, Sommerlad A, Orgeta V, et al. Dementia prevention, intervention, and care. Lancet 2017;390:2673-734.

18. Bullain SS, Corrada MM. Dementia in the oldest old. Continuum (Minneap Minn) 2013;19:457-69.

19. Lin FR, Yaffe K, Xia J, et al. Hearing loss and cognitive decline in older adults. JAMA Intern Med 2013;173:293-9.

20. Allen NH, Burns A, Newton V, et al. The effects of improving hearing in dementia. Age Ageing 2003;32:189-93.

21. Ruxton K, Woodman RJ, Mangoni AA. Drugs with anticholinergic effects and cognitive impairment, falls and allcause mortality in older adults: a systematic review and meta-analysis. $\mathrm{Br} \mathrm{J}$ Clin Pharmacol 2015;80:209-20.

22. Bullock R, Bergman H, Touchon J, et al. Effect of age on response to rivastigmine or donepezil in patients with Alzheimer's disease. Curr Med Res Opin 2006;22:483-94.

23. Boccardi V, Baroni M, Smirne N, et al. Short-term response is not predictive of long-term response to acetylcholinesterase inhibitors in old age subjects with Alzheimer's disease: a "real world" study. JAD 2017;56:239-48. 